\title{
Structure Determination of Isolated Metal Clusters via Far-Infrared Spectroscopy
}

\author{
André Fielicke, ${ }^{1}$ Andrei Kirilyuk, ${ }^{2}$ Christian Ratsch, ${ }^{3,4}$ Jörg Behler, ${ }^{3}$ Matthias Scheffler, ${ }^{3}$ \\ Gert von Helden, ${ }^{3}$ and Gerard Meijer ${ }^{3}$ \\ ${ }^{1}$ FOM Institute for Plasma Physics Rijnhuizen, Edisonbaan 14, NL-3439 MN Nieuwegein, The Netherlands \\ ${ }^{2}$ NSRIM Institute, University of Nijmegen, Toernooiveld 1, NL-6525 ED Nijmegen, The Netherlands \\ ${ }^{3}$ Fritz-Haber-Institut der Max-Planck-Gesellschaft, Faradayweg 4-6, D-14195 Berlin, Germany \\ ${ }^{4}$ Department of Mathematics, UCLA, Los Angeles, California 90095-1555, USA
}

(Received 19 March 2004; published 7 July 2004)

\begin{abstract}
We present a new method for the size selective structure determination of small isolated metal clusters in the gas phase. The technique is applied to cationic vanadium clusters containing 6 to 23 atoms, whose far infrared absorption spectra are measured in the $140-450 \mathrm{~cm}^{-1}$ spectral range. The spectra are unique for each cluster size and are true fingerprints of the cluster's structure. By comparing the experimental spectra to spectra obtained from density-functional theory, the geometric cluster structure can be identified.
\end{abstract}

DOI: 10.1103/PhysRevLett.93.023401

PACS numbers: $36.40 . \mathrm{Mr}, 61.46 .+\mathrm{w}$

Small metal particles are rapidly gaining importance in nanostructured material science and catalysis [1]. The geometric and electronic structures of such small clusters differ from those of bulk material, and they can have surprising properties, as, for example, demonstrated by the catalytic activity of gold nanoparticles [2]. In the cluster size range of up to several hundred atoms, the physical and chemical properties can change drastically by the addition of a single atom to the cluster [3-5]. This strong cluster size dependence is not easily predictable by, for example, scaling laws. It is thus important to gain fundamental understanding on the structures and properties of small metal clusters. The experimental determination of metal cluster structures in the gas phase is difficult, and only very recently have structures of gold and silver cluster ions been deduced from experimental collision cross sections and comparison to theory [6]. Information on the electronic structure of metal clusters can be obtained from photoelectron spectroscopy as well as from optical spectroscopy. Sometimes, such data can also be used to gain information on the geometric structure of the cluster.

A preferred method to obtain information on structure and bonding is vibrational spectroscopy. Unfortunately, in most cases, isolated clusters can be studied only in molecular beams or special (ion) traps, and the low particle density rules out direct absorption measurements. Furthermore, clusters are often produced in broad size distributions, making size selectivity essential. To overcome such problems, mass selected clusters can be embedded and accumulated in rare gas matrices. Raman spectra have been measured on embedded transition metal trimers, and in a few cases even spectra of bigger clusters $\left(\mathrm{Ta}_{4}, \mathrm{Sc}_{4}, \mathrm{Ag}_{5}, \mathrm{Ag}_{7}, \mathrm{Ag}_{9}\right)$ have been obtained $[7,8]$. In those experiments, interactions with the rare gas matrix may induce shifts of the absorption lines, and, additionally, it cannot be excluded that structural changes during the deposition occur. For measurements in the gas phase, the absorption of photons can be detected by monitoring the dissociation of weakly bound cluster complexes. Monitoring the dissociation yield as a function of photon energy then gives the absorption spectrum. Although such techniques are used to measure UV, optical, and near infrared absorption spectra of vanadium $[9,10]$ and other transition metal clusters [11-14], the lack of easily accessible intense and tuneable far infrared sources has prohibited so far the use of this method for the direct measurement of metal cluster vibrational spectra.

Here, we demonstrate infrared multiple photon dissociation (IR-MPD) spectroscopy on metal cluster complexes with rare gas atoms, and we discuss the thus obtained far infrared (FIR) absorption spectra of vanadium cluster cations. Our experiments are performed in a molecular beam setup coupled to the beam line of the Free Electron Laser for Infrared eXperiments (FELIX) [15] user facility at the FOM institute. All spectral data presented in this Letter have been accumulated within six FELIX shifts $(\approx 50 \mathrm{~h}$ of beam time). A scheme of the experiment is shown in Fig. 1. The cluster cations are produced by ablating the metal with the second harmonic output $(532 \mathrm{~nm}, \sim 10 \mathrm{~mJ})$ of a pulsed $\mathrm{Nd}$-doped yttrium aluminum garnet (Nd:YAG) laser and quenching the plasma with a short pulse of a gas mixture containing $0.5 \%$ argon in helium. Complexes with Ar are formed in a copper channel that is maintained at $\sim 80 \mathrm{~K}$ by contact with a liquid nitrogen filled reservoir. After expansion into vacuum the cluster distribution in the molecular beam is analyzed using a reflectron time-of-flight mass spectrometer. The molecular beam is overlapped with a counterpropagating intense infrared laser beam, delivered by FELIX. FELIX can emit tuneable pulsed radiation in the $40-2000 \mathrm{~cm}^{-1}$ range and is here scanned throughout the $140-580 \mathrm{~cm}^{-1}$ spectral region to excite 


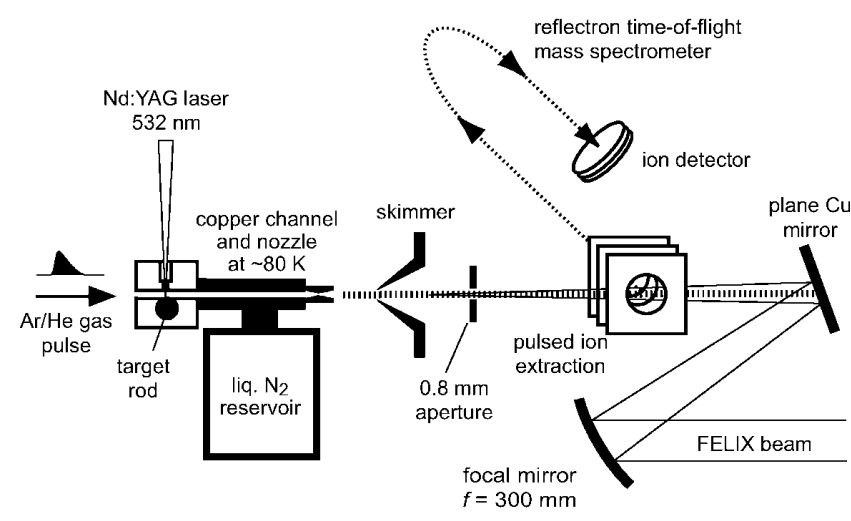

FIG. 1. Scheme of the experimental setup for IR multiple photon dissociation of metal cluster-rare gas complexes. The experiments are performed on metal cluster cations directly emitted from the cluster source.

the complexes of vanadium cluster cations with one or two argon atoms. If the laser light is resonant with an IR active mode of a cluster, one or more photons can be absorbed by the cluster. If this leads to dissociation of the argon complexes, their mass spectrometric intensity will be decreased (see Fig. 2). IR depletion spectra are constructed by recording the ion intensities of the argon complexes as a function of the FELIX frequency. The use of mass spectrometric detection of the IR induced dissociation allows for cluster size specific measurement of the IR absorption spectra. Using the IR-MPD technique, IR spectra of metal cluster complexes have been measured in the mid IR using resonant excitation of internal ligand vibrations of $\mathrm{NH}_{3}$ or $\mathrm{CO}$ [16]. In those experiments, however, no information on the internal cluster structure could be obtained.

We also performed density-functional theory (DFT) calculations to identify the structural and vibrational

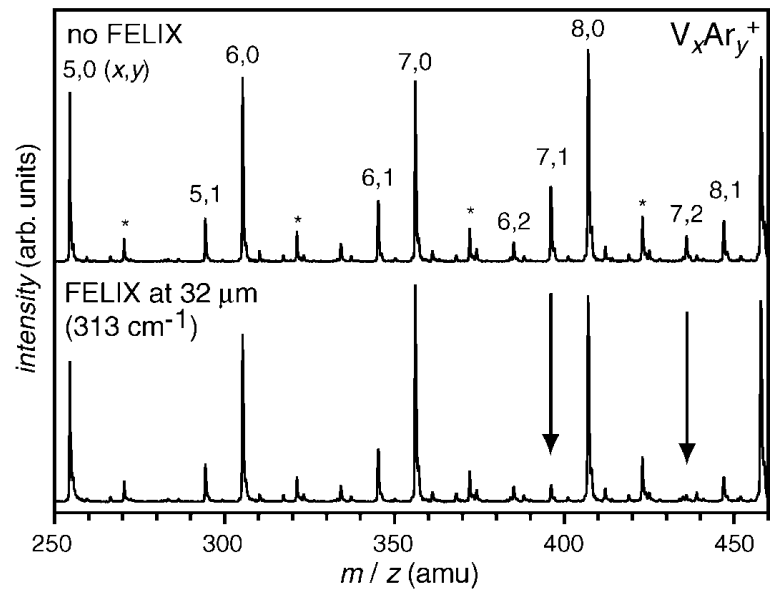

FIG. 2. Part of mass spectra of vanadium cluster cations and their argon adducts. The arrows in the lower mass spectrum show the depleted signals for $\mathrm{V}_{7} \mathrm{Ar}^{+}$and $\mathrm{V}_{7} \mathrm{Ar}_{2}{ }^{+}$, which have an intense resonance around $313 \mathrm{~cm}^{-1}$. (The asterisks mark oxide contaminations $\mathrm{V}_{n} \mathrm{O}^{+}$.) properties for the vanadium cluster. We employed the $\mathrm{DMol}^{3}$ code [17], which uses a basis set that consists of numerical atomic orbitals. The generalized gradient approximation in the parametrization of Perdew, Burke, and Ernzerhof [18] was used for the exchange-correlation functional. Many different geometries and spin states were tested in the calculations. The convergence of all results was tested carefully and the structures were fully optimized. The frequencies are obtained by diagonalizing the force constant matrix, which is obtained by displacing each atom in the cluster in all different directions.

In the experiments presented here, we measure the FIR depletion spectra of the rare gas complexes of the metal cluster cations. The rare gas atom interacts only weakly with the metal cluster. Therefore, we can identify the measured spectra with those of the bare metal cluster cations. The measured depletion signals are converted to absorption cross sections $\sigma(\nu)$ and normalized for variations of the laser power $P(\nu)$ over the tuning range using

$$
\sigma(\nu)=\ln \left[I_{0} / I(\nu)\right] / P(\nu)
$$

where $I(\nu)$ and $I_{0}$ are the intensities of a certain $\mathrm{V}_{n} \mathrm{Ar}^{+}$ complex with and without FELIX irradiation, respectively. This procedure assumes a one photon absorption process, and because not only the IR power but also the spectral width and shape of the focused beam is changing with the wavelength, it is not at all clear if this simple normalization suffices. It has been shown, however, that this approach can yield spectra that are very similar to one photon absorption spectra [19]. The thus obtained FIR absorption spectra for vanadium cluster cations containing 6 to 23 atoms are presented in Fig. 3. The spectra are selected to show only the range from 140 to $450 \mathrm{~cm}^{-1}$, as no signals have been found at a higher photon energy. The width of the observed peaks can be as narrow as $5 \mathrm{~cm}^{-1}$, and is in all cases wider than the bandwidth of the FIR excitation laser, which is typically $1 \%$ of its central frequency. Relative intensities of peaks that are in the vicinity of each other are expected to be accurate within $20 \%$. However, it should be kept in mind that over the whole range the photon energy changes by more than a factor of 3 , and therefore the relative intensities of peaks at the extremes of the range can be off considerably more.

For the smallest cluster shown, $\mathrm{V}_{6}{ }^{+}$, two resonances are observed, where the feature at higher photon energy is broad and might consist of several unresolved peaks. Around $250 \mathrm{~cm}^{-1}$ increased noise is observed in this as well as in some other spectra due to a reduced laser intensity in the 40-45 $\mu \mathrm{m}$ region. For $\mathrm{V}_{7}{ }^{+}$, again two peaks are observed and $\mathrm{V}_{8}{ }^{+}$shows the presence of five narrow peaks. The cluster with the richest spectral structure is $\mathrm{V}_{14}{ }^{+}$. There, at least ten well resolved resonances are observed. Interestingly, the two clusters adjacent to this one, $\mathrm{V}_{13}{ }^{+}$and $\mathrm{V}_{15}{ }^{+}$, show the presence of only 


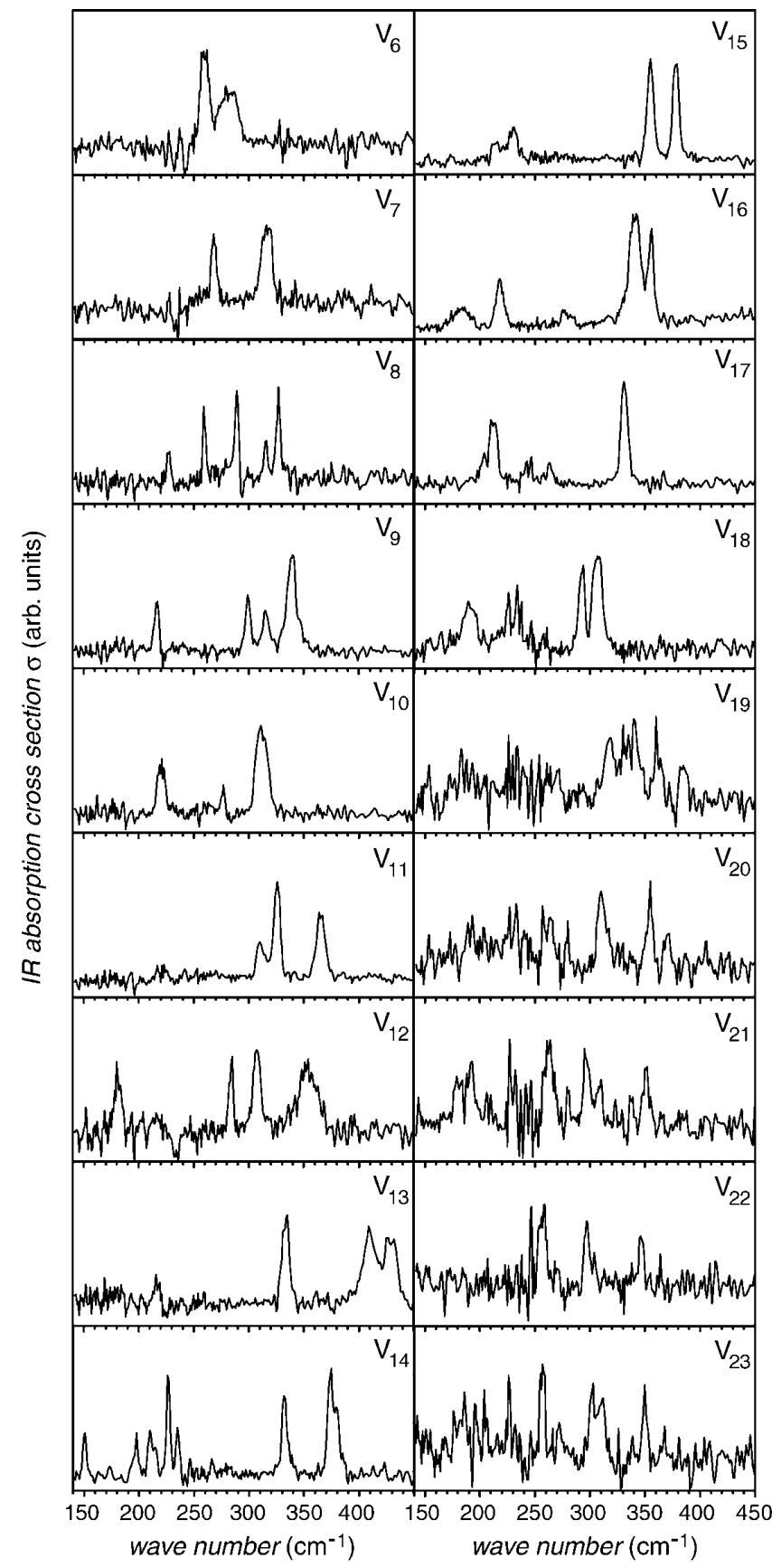

FIG. 3. Far infrared multiple photon dissociation spectra of the argon complexes of vanadium cluster cations $\mathrm{V}_{n} \mathrm{Ar}_{1,2}{ }^{+}$. The spectra are measured in the range of $140-580 \mathrm{~cm}^{-1}$, but only the range up to $450 \mathrm{~cm}^{-1}$, where signals have been found, is displayed. For $n=6$ and 7 the spectra are obtained from the complexes with two Ar atoms, and for all the others with one Ar atom. A maximum shift of about $2 \mathrm{~cm}^{-1}$ is observed for the IR active modes in $\mathrm{V}_{8}{ }^{+}$when comparing the depletion spectra of $\mathrm{V}_{8} \mathrm{Ar}_{1}{ }^{+}$and $\mathrm{V}_{8} \mathrm{Ar}_{2}{ }^{+}$.

about four peaks, indicative of structures with higher symmetry. For clusters with 20 atoms or more, the signal to noise ratio slowly deteriorates; nonetheless, well resolved resonances are still observed. Most importantly, when looking at the whole set of spectra it becomes clear that the experimental spectra depend sensitively on the cluster size and that each cluster has its individual "fingerprint" spectrum. IR-MPD spectra for smaller clusters $\mathrm{V}_{n}{ }^{+}(n=3-5)$ are not shown. For those clusters we observe differences in the dissociation spectra depending on the number of attached Ar atoms, and the low density of vibrational states of these small clusters might affect the efficiency of the IR-MPD process. For this reason, the obtained IR-MPD spectra can differ more from the IR absorption spectra than in the case of the larger clusters discussed in this Letter.

The bulk structure of vanadium is body centered cubic (bcc) with a shortest V-V distance of $2.62 \AA$. The two optically active phonon modes are at $\sim 165 \mathrm{~cm}^{-1}$ (transversal) and $230 \mathrm{~cm}^{-1}$ (longitudinal) [20]. All our spectra have signals in this range as well as up to a factor of 2 higher frequency. Structural properties of small vanadium cluster cations $\mathrm{V}_{n}{ }^{+}(n=3-5)$ have been derived on the basis of their electronic excitation spectra [9] and for clusters containing up to 100 atoms using anion photoelectron spectroscopy [21,22]. In the electronic structure of anionic vanadium clusters similarities to the bulk have been found starting with the 13 atom cluster, where a band appears that has been assigned to a surface feature. A second bulklike feature is evolving starting with the 17 atom cluster. The interpretation of Wu et al. is that already $\mathrm{V}_{17}{ }^{-}$possesses an inner structure similar to that of the bulk [22].

To obtain structural information, the experimental spectra can be compared to results from quantum chemical calculations. For this, we have chosen $\mathrm{V}_{8}{ }^{+}$, as it is one of the clusters that shows a very detail-rich experimental spectrum and yet it is still small enough to allow for accurate and reliable calculations. Figure 4 shows for $\mathrm{V}_{8}{ }^{+}$the comparison of the experimental spectrum with calculated IR absorption spectra of the four lowest energy isomers found. The calculated spectra depend very sensitively on the geometric and electronic structure of the cluster. The lowest energy structure $A$ is a tetragonal bipyramid with two additional atoms on two faces (the two neighboring atoms most to the left in the figure). The spin state is a doublet. Structure B is a doublet as well and can be described as a distorted hexagonal bipyramid where the atoms forming the hexagon are not all in one plane. Structure $C$ is very close to structure $A$, however, with a quartet spin state. Structure D has the least symmetry of the four. The relative energies are given in the figure. The octahedral subunit in $A$ and $C$ is an element of the bcc structure of vanadium metal. The experimental spectrum shows the presence of five resolved lines. A visual inspection shows that the spectra of structure $B$ and $D$ are incompatible with the experimental findings. The spectra of structure A and C, on the other hand, match the experimental spectrum quite well, with the spectrum of $\mathrm{C}$ arguably being the better match. Previous theoretical investigations already predicted a structure similar to $\mathrm{A}$ and $\mathrm{C}$ as the lowest energy isomer 


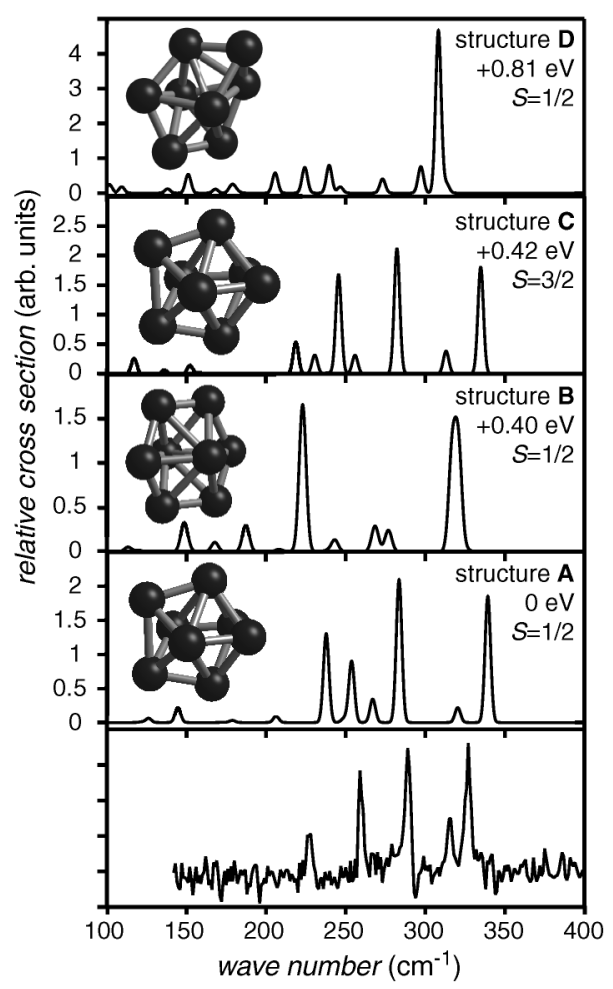

FIG. 4. Comparison of experimental IR spectra for $\mathrm{V}_{8}{ }^{+}$with calculated IR absorption spectra of some isomers. The calculated frequency spectrum is scaled by a constant factor of 0.87 , which was obtained by comparison of the experimental dimer vibrational frequency with that calculated with DFT. The spectra are folded with a Gaussian line shape function of $4 \mathrm{~cm}^{-1}$ width (FWHM).

of $\mathrm{V}_{8}{ }^{+}$[23]. The good match between the calculated spectra of $A$ and $C$ with the experimental spectrum demonstrates that this measurement strategy allows the determination of the geometric structure of the cluster.

In conclusion, we have shown that multiple photon dissociation using an intense far infrared laser source can be applied to obtain unique spectroscopic information on metal particles in molecular beams. This method allows the measurement of IR absorption spectra for small metal clusters, and in combination with theory it can be used to determine the cluster structures. The availability of these new experimental data on the vibrational properties of metal clusters is expected to stimulate further calculations and developments of theoretical methods leading to an improved understanding of the structures and dynamics of these important species.

This work is part of the research program of the "Stichting voor Fundamenteel Onderzoek der Materie" (FOM), which is supported financially by the "Nederlandse organisatie voor Wetenschappelijk Onderzoek"
(NWO). Financial support from the EU IHP Research Training Network (Delayed Ionisation and Competing Cooling Mechanisms in Atomic Clusters) and from the Deutsche Forschungsgemeinschaft is gratefully acknowledged. We are grateful to David M. Rayner for stimulating discussions and for providing us with an initial version of the $80 \mathrm{~K}$ source channel.

[1] A. T. Bell, Science 299, 1688 (2003).

[2] M. Valden, X. Lai, and D.W. Goodman, Science 281, 1647 (1998).

[3] W. A. de Heer, Rev. Mod. Phys. 65, 611 (1993).

[4] M. B. Knickelbein, Annu. Rev. Phys. Chem. 50, 79 (1999).

[5] H. Häkkinen et al., Angew. Chem., Int. Ed. Engl. 42, 1297 (2003).

[6] P. Weis, T. Bierweiler, S. Gilb, and M. M. Kappes, Chem. Phys. Lett. 355, 355 (2002); S. Gilb et al., J. Chem. Phys. 116, 4094 (2002); F. Furche et al., J. Chem. Phys. 117, 6982 (2002).

[7] K. A. Bosnick et al., J. Chem. Phys. 111, 8867 (1999).

[8] J. R. Lombardi and B. Davis, Chem. Rev. 102, 2431 (2002); B. Zhao, H. Lu, J. Jules, and J. R. Lombardi, Chem. Phys. Lett. 362, 90 (2002).

[9] S. Minemoto, A. Terasaki, H. Imoto, and T. Kondow, J. Chem. Phys. 109, 9737 (1998); S. Minemoto, A. Terasaki, and T. Kondow, J. Electron Spectrosc. Relat. Phenom. 106, 171 (2000).

[10] J. M. Antonietti, A. Châtelain, and S. Fedrigo, J. Chem. Phys. 114, 2981 (2001).

[11] W. J. C. Menezes and M. B. Knickelbein, J. Chem. Phys. 98, 1856 (1993); M. B. Knickelbein, J. Chem. Phys. 100, 4729 (1994).

[12] B. A. Collings et al., J. Chem. Phys. 101, 3506 (1994).

[13] S. Minemoto, A. Terasaki, and T. Kondow, J. Chem. Phys. 104, 5770 (1996).

[14] A. Schweizer et al., J. Chem. Phys. 119, 3699 (2003).

[15] D. Oepts, A.F.G. van der Meer, and P.W. van Amersfoort, Infrared Phys. Technol. 36, 297 (1995).

[16] B. Simard et al., Chem. Phys. Lett. 357, 195 (2002); A. Fielicke et al., J. Am. Chem. Soc. 125, 11184 (2003).

[17] B. Delley, J. Chem. Phys. 92, 508 (1990).

[18] J. P. Perdew, K. Burke, and M. Ernzerhof, Phys. Rev. Lett. 77, 3865 (1996).

[19] J. Oomens, A. G. G. M. Tielens, B. Sartakov, G. von Helden, and G. Meijer, Astrophys. J. 591, 968 (2003).

[20] V.F. Sears, E.C. Svensson, and B. M. Powell, Can. J. Phys. 73, 726 (1995).

[21] M. Iseda et al., J. Chem. Phys. 106, 2182 (1997).

[22] H. Wu, S. R. Desai, and L.-S. Wang, Phys. Rev. Lett. 77, 2436 (1996).

[23] X. Wu and A. K. Ray, J. Chem. Phys. 110, 2437 (1999). 